\title{
Análisis de propulsión iónica en condensadores asimétricos
}

\author{
Analysis of Ion Propulsion in Asymmetric Capacitors
}

\author{
Fredy Yamith Cubides Alfonso ${ }^{\mathrm{a}^{*}}$ \\ David Leonardo Lancheros Quiroga ${ }^{\mathrm{b}}$ \\ César Alexander Chacón-Cardona ${ }^{\mathrm{c}}$
}

Recepción: 22-ene-2016

Aceptación: 13-jun-2016

\section{Resumen}

Presenta una nueva metodología para el estudio del comportamiento en condensadores asimétricos del fenómeno de levitación electromagnética, sometido a altas tensiones (AC y DC).

Se realizaron modelos experimentales para un análisis cinemático y simulaciones para observar el campo eléctrico y magnético, y, por último, se hicieron estudios de termografía de los condensadores asimétricos, con el fin de realizar un análisis cuantitativo y cualitativo del fenómeno de levitación electromagnética, comparando el comportamiento que se presenta con cada tipo de señal (AC y DC). Como resultado, el condensador asimétrico sometido a $30 \mathrm{kV}$ en corriente directa presentó una levitación estable, en comparación con la señal en corriente alterna.

Palabras clave: Alta tensión, Arco eléctrico, Campo eléctrico, Capacitor asimétrico, Ionización.

\begin{abstract}
This paper shows a new methodology for the study of the behaviour of asymmetrical capacitors subjected to high voltage (AC and DC).

Experimental models for a kinematic analysis and simulations to observe the electric and magnetic field are shown. Additionally, in order to conduct a quantitative and qualitative analysis of the electromagnetic levitation phenomenon, thermography studies were performed by comparing the behaviours resulted from each signal type (AC and DC). The results showed the asymmetric capacitor connected to $30 \mathrm{kV}$ in direct current presented a stable levitation in comparison with the AC current signal.
\end{abstract}

Key words: High voltage, Electric arc, Electric field, Asymmetric capacitor, Ionization.

\footnotetext{
${ }^{a}$ Estudiante de Ingeniería Eléctrica, Tecnólogo en electricidad, de la Universidad Distrital Francisco José de Caldas, Facultad Tecnológica, Integrante del Grupo de Investigación AstroUD Sabio Caldas.

*Autor de correspondencia: freddy.cubides@gmail.com

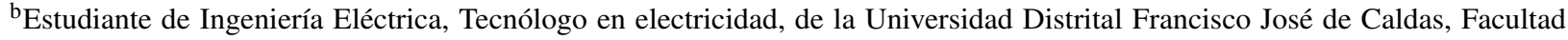
Tecnológica, e integrante del grupo de investigación AstroUD Sabio Caldas.

${ }^{\mathrm{c}}$ Físico, magíster en ciencias físicas, doctor en ciencias físicas, de la Universidad Nacional de Colombia; investigador científico, docente de la Universidad Distrital Francisco José de Caldas e integrante del Grupo de Investigación AstroUD Sabio Caldas.
} 


\section{Introducción}

El Condensador asimétrico, también conocido como ACT (Asymmetrical Capacitor Thruster) o lifter, es un condensador cuyos electrodos son distintos entre sí; de geometrías similares, pero diferentes en cuanto a la altura [1] (ver figura1); al someter este condensador a un alto potencial eléctrico se presenta un fenómeno de levitación, conocido como efecto Biefiel-Brown, descubierto en 1922 [2].
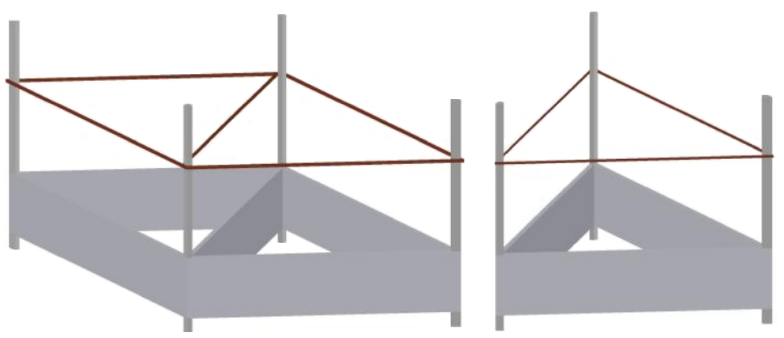

Figura 1. Modelos de capacitor asimétrico

En un comienzo, este principio fue patentado en los Estados Unidos como "Transductor electrocinético", con la patente 3018394, que describe dos electrodos de diferentes dimensiones inmersos en un medio dieléctrico (aire), sometidos a un alto potencial que provoca que los electrones circundantes se dirijan al terminal positivo, mientras las moléculas cargadas positivamente se dirigen al terminal negativo, de modo que se genera un viento que empuja al transductor, generando energía mecánica y provocando la levitación [3].

Son múltiples los estudios que se han desarrollado acerca del condensador asimétrico, con el propósito de explicar el comportamiento de levitación, pero estos no incluyen simulaciones que permitan observar las líneas de campo eléctrico y magnético. En la literatura tampoco se encuentran investigaciones del fenómeno de levitación en corriente alterna; este fenómeno físico es una alternativa para el movimiento y propulsión de objetos, así como lo hace la tecnología de levitación magnética, empleada para impulsar el tren bala de Japón [4].

El aprovechamiento de este fenómeno serviría para generar avances e investigaciones tecnológicas, como lo puede ser la construcción e implementación de pequeñas fuentes de alta tensión, estudios de apantallamiento para un posible control electrónico e investigación de materiales aislantes y livianos.
En el análisis realizado por Zhao (2009) se expone un algoritmo numérico que se basa en métodos de elementos finitos y de contorno, para hacer una simulación del flujo de aire y descarga por efecto corona, teniendo presente que el flujo de aire y el efecto corona no son separables, como normalmente no se hace por la despreciable cantidad de densidad de corriente. Los resultados de la simulación permiten predecir el flujo de aire y sus efectos en la descarga por efecto corona [5].

En el trabajo realizado por Martins y Pinheiro (2011) se realizó un estudio con el propósito de comprender la propulsión presentada en un efecto corona que ocurre dentro de gas de nitrógeno, y se hicieron interpretaciones físicas de los resultados, sugiriendo posibles razones de la naturaleza del capacitor asimétrico [6].

En el trabajo de Rincón y Amaya (2012) fueron ideados experimentos nuevos, como encerrar el capacitor asimétrico en un recipiente con humo para visualizar el efecto del ozono generado, comprobando que el prototipo opera en forma equivalente a un separador electrostático convencional, eliminando partículas sólidas de un gas encerrado en el recipiente, se hicieron pruebas termográficas y se arrojaron pequeñas esferas de poliestireno para visualizar la dirección del gas ionizado alrededor del capacitor [7]. No se hicieron mediciones de fuerza y tampoco ensayos en corriente alterna.

En el ensayo experimental realizado por Primas, Malík, Jasíková y Kopecký (2012) se aplicó un alto voltaje en corriente continua a un condensador asimétrico, el cual generó una fuerza mecánica que afectó todo el condensador, causada por el movimiento de iones generado en torno al menor de los dos electrodos y su posterior interacción con el medio circundante. Se calentó un electrodo del capacitor asimétrico con la hipótesis de que se facilitaría el proceso de ionización del aire, lo que presentó un aumento en la propulsión generada por el condensador y registró una mayor fuerza; para el registro de la fuerza utilizaron una balanza [8].

En el estudio de Cattani y Vannucci (2014) se diseñó un capacitor asimétrico con una geometría triangular basada en las bisectrices de un triángulo equilátero y una fuente de alta tensión, utilizando un flyback. Se realizó una estimación de la fuerza debido a la corriente y la tensión aplicada al dispositivo, 
pero no se llevó a cabo una medición experimental de la fuerza; el estudio se realizó en corriente directa [9].

En el estudio de Howell (2014) se realizó un análisis para la determinación de propiedades electromagnéticas alrededor del dispositivo; el dispositivo fue ideado para realizar un movimiento rotatorio horizontal. El estudio consistió en ver la reacción electromagnética cerca del dispositivo al cambiar los valores de alimentación en corriente directa [10].

De acuerdo con los estudios realizados, el presente artículo se dirige a mostrar los resultados experimentales en corriente alterna y directa, en tres casos:

\section{- Estudio cinemático}

Al presenciar el fenómeno de levitación del condensador asimétrico, se pueden obtener curvas de desplazamiento, velocidad y aceleración por medio del programa computacional Tracker, que modela curvas cinemáticas a partir de videos, con la finalidad de hallar el valor de la fuerza que necesita el condensador asimétrico para levitar (este programa computacional es de uso libre).

\section{- Visualización del campo eléctrico y magnético}

Para ilustrar la naturaleza de los campos asociados a los condensadores asimétricos se hicieron modelos y simulaciones en el programa computacional Comsol Multiphysics (licencia adquirida por la Universidad Distrital Francisco José de Caldas), el cual muestra las líneas de campo eléctrico y magnético, permitiendo una visión a fondo de la física electromagnética asociada al experimento.

\section{- Estudio termográfico}

La cámara de termografía FLIR permite observar la distribución y variación de temperatura de cada condensador asimétrico cuando se somete a cada tipo de señal de corriente.

El estudio termográfico ofrece una descripción del comportamiento físico del fenómeno, al incluir la medición de variables termodinámicas como la temperatura.

\section{Hipótesis acerca del fenómeno físico}

En el desarrollo de ensayos experimentales se han propuesto teorías que intentan explicar el comportamiento de levitación de los condensadores asimétricos. Las hipótesis que enuncian dicho comportamiento son las siguientes:

\subsection{Materiales ablativos}

El material ablativo es usualmente usado en misiones espaciales en las que la coraza del transbordador se destruye a medida que ingresa a la atmósfera [11]. En el capacitor asimétrico, al tener una diferencia de potencial muy alta, las partículas se cargan eléctricamente y viajan a alta velocidad $(930 \mathrm{~m} / \mathrm{s}$ apox.) directamente de un electrodo a otro; ambos electrodos presentan una temperatura de alrededor de 2.326 ř $\mathrm{C}$ y, teóricamente, con el paso de las partículas presentaría una deformación (ablación), y a medida que se presenta la levitación los electrodos se destruyen [2], al igual que la coraza del transbordador al ingresar a la Tierra.

\subsection{Fuerzas electrostáticas}

Haciendo el ensayo del condensador asimétrico en una cámara de vacío, los electrodos del condensador podrían cargarse de manera contraria a la carga de objetos, que podrían estar fuera de la cámara, o las paredes de la misma cámara de vacío, generando una fuerza de atracción o repulsión que explicaría el movimiento del capacitor [2].

\subsection{Ionización del aire}

En una cámara de vacío se brinda energía al condensador asimétrico, y hay una filtración de corriente de una placa a la otra, los iones transfieren el momentum (cantidad de movimiento) [12] a una placa del condensador, pero no hay un efecto neto. Mientras que en presencia de atmósfera se presenta un empuje debido a que el flujo de corrientes de una placa a la otra, por medio de las líneas de campo eléctrico, choca con las partículas del aire, transfiriendo así el momentum al aire y provocando un empuje. Asumiendo este criterio puede estimarse la fuerza, como se muestra en la Ec. 1, en donde la fuerza depende de la carga y el campo [2].

$$
F=q E
$$


Donde:

$F$ : fuerza eléctrica $[\mathrm{N}]$

$q$ : carga eléctrica $[\mathrm{C}]$

$E$ : campo eléctrico $[\mathrm{V} / \mathrm{m}]$

\section{Metodología}

A continuación se describe el procedimiento desarrollado para analizar el comportamiento del condensador asimétrico, sometido a altas tensiones en corriente alterna y directa.

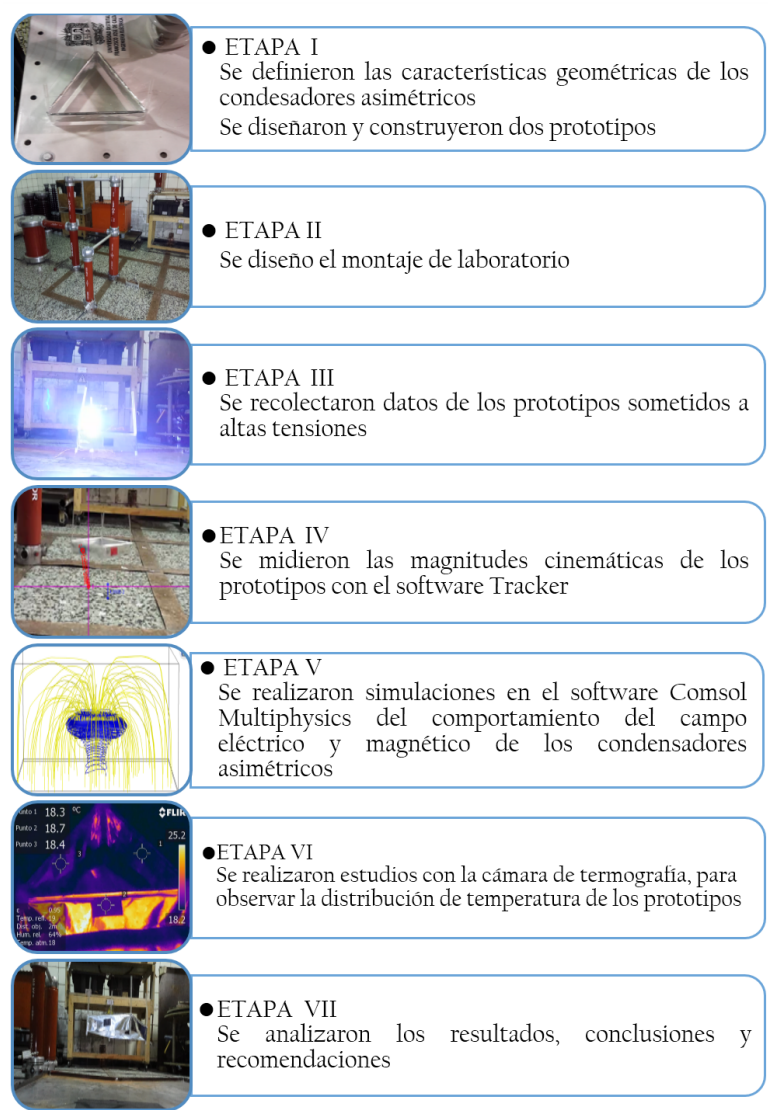

Figura 2. Metodología

\section{Criterios de diseño y construcción de condensador asimétrico}

Se definieron las características del diseño y se presentaron dos configuraciones para el condensador asimétrico; además, se optimizó el peso de cada prototipo en el proceso de construcción.

\subsection{Diseño de los prototipos}

Se consideraron dos configuraciones geométricas; para el condensador asimétrico 1 (ver figura 3) se diseñó una geometría con base en un trián- gulo equilátero, y el condensador asimétrico 2 (ver figura4) fue una variante del 1 , con una configuración de dos triángulos equiláteros.

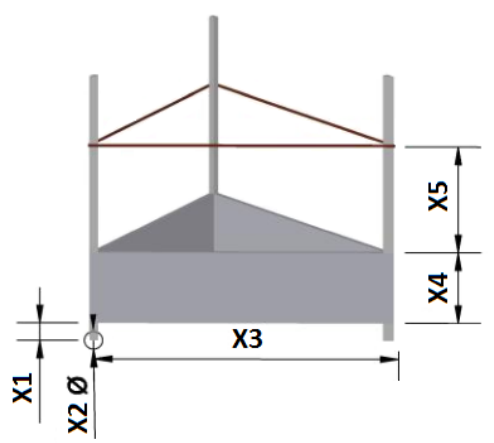

Figura 3. Condensador asimétrico 1, configuración de un triángulo equilátero

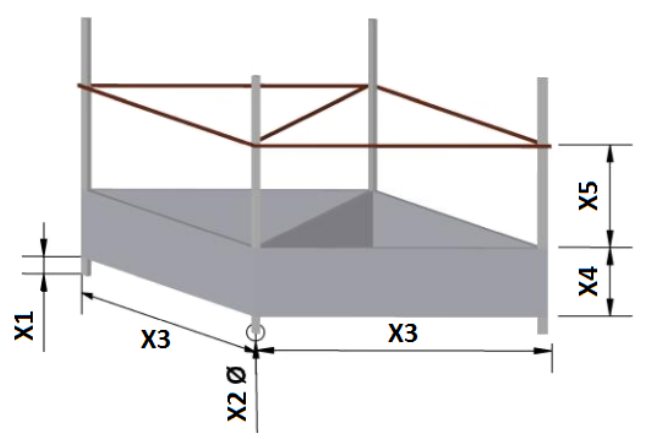

Figura 4. Condensador asimétrico 2, configuración de dos triángulos equiláteros

En la tabla 1 se presentan las dimensiones de los condensadores.

Tabla 1. Dimensiones de los condensadores asimétricos.

\begin{tabular}{ccccc}
\hline \multicolumn{5}{c}{ Distancias [mm] } \\
\hline $\mathrm{X} 1$ & $\mathrm{X} 2$ & $\mathrm{X} 3$ & $\mathrm{X} 4$ & $\mathrm{X} 5$ \\
\hline $10 \pm 0.1$ & $5 \pm 0.1$ & $150 \pm 0.1$ & $40 \pm 0.1$ & $60 \pm 0.1$ \\
\hline
\end{tabular}

\subsection{Construcción de los prototipos}

Para obtener un prototipo de condensador asimétrico liviano se usaron los siguientes materiales:

- Electrodo inferior: papel aluminio

- Electrodo superior: cable de cobre (30 AWG)

- Soportes: pajillas de polipropileno (diámetro 5 $\pm 1 \mathrm{~mm})$

De acuerdo con los diseños planteados, se obtuvieron los siguientes prototipos:

- Condensador asimétrico 1 (ver figura 5), $m=$ $3.22 \pm 0.01 \mathrm{~g}$. 


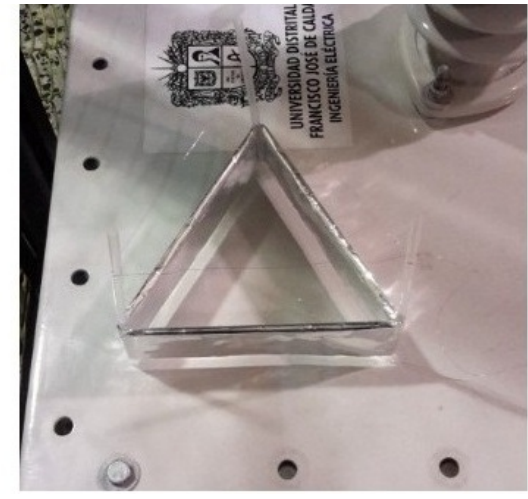

Figura 5. Condensador asimétrico 1

- Condensador asimétrico 2 (ver figura 6), $m=$ $4.78 \pm 0.01 \mathrm{~g}$.

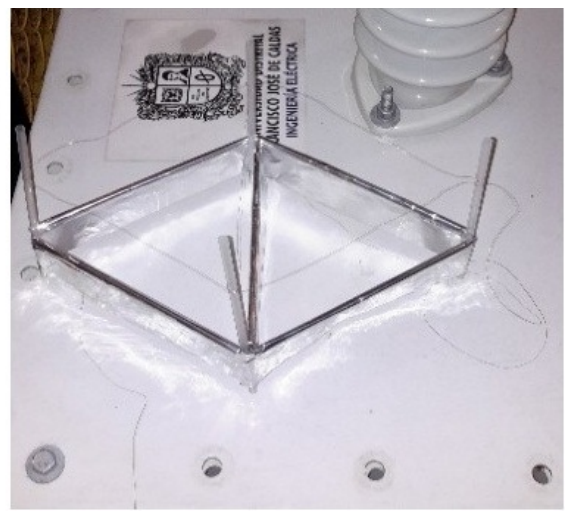

Figura 6. Condensador asimétrico 1

\section{Montaje}

El montaje se realizó en el Laboratorio de Alta Tensión, de la Facultad Tecnológica de la Universidad Distrital Francisco José de Caldas; los equipos que se utilizaron se encuentran en las tablas 2 y 3 .

Tabla 2. Equipos de alta tensión.

\begin{tabular}{lc}
\hline Equipos & Características \\
Aislador & $140 \mathrm{kV}$ \\
Condensador & $140 \mathrm{kV} \cdot 25 \mathrm{nF}$ \\
Diodo & $140 \mathrm{kV} \cdot 100 \mathrm{k} \Omega$ \\
Divisor resitivo & $140 \mathrm{kV} \cdot 280 \mathrm{M} \Omega$ \\
Módulo de control HV 9103 & $5 \mathrm{kVA} \cdot 0-230 \mathrm{~V}$ \\
Transformador & $5 \mathrm{kVA} \cdot 220-100 \mathrm{kV}$ \\
\hline
\end{tabular}

Para realizar las mediciones en alta tensión se acopló una sonda al divisor resistivo y se conectó al multímetro Fluke 289, teniendo una relación de transformación de 7029 (ver tabla 3).

Tabla 3. Multímetro Fluke 289.

\begin{tabular}{lccc}
\hline Señal & Rango & Resolución & Exactitud \\
DC & 5 & 0.0001 & $0.025 \%+2$ \\
AC $45-65 \mathrm{~Hz}$ & 5 & 0.0001 & $0.3 \%+25$ \\
\hline
\end{tabular}

A continuación se observan los montajes empleados en corriente alterna (ver figura 7) y directa (ver figura 8) para cada condensador asimétrico; el electrodo inferior se encuentra conectado a tierra, y el superior, al terminal positivo o fase de la fuente.

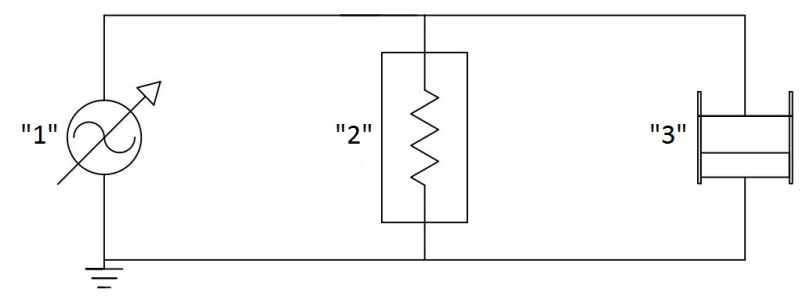

Figura 7. Montaje de laboratorio para corriente alterna

Donde

$$
\begin{aligned}
& 1 \text { : Fuente } 220 \text { - } 100 \mathrm{kV} \\
& 2 \text { : Divisor resistivo } 280 \mathrm{M} \Omega \\
& 3 \text { : Condensador asimétrico }
\end{aligned}
$$

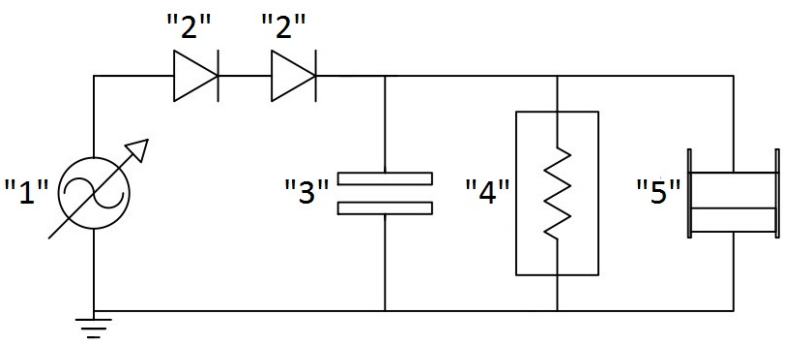

Figura 8. Montaje de laboratorio para corriente directa

Donde

1 : Fuente 220 - $100 \mathrm{kV}$

2 : Diodo $100 \mathrm{k} \Omega$

3 : Condensador $25 \mathrm{nF}$

4 : Divisor resistivo $280 \mathrm{M} \Omega$

5 : Condensador asimétrico

\section{Ensayos preliminares}

Inicialmente se realizaron ensayos con los dos montajes, sin embargo, no se evidenció estabilidad aceptable de ninguno de los prototipos, debido a que el sistema de puesta a tierra no era homogéneo, por lo cual se modificó, colocando una lámina metálica, como se puede observar en la figura 9 . 


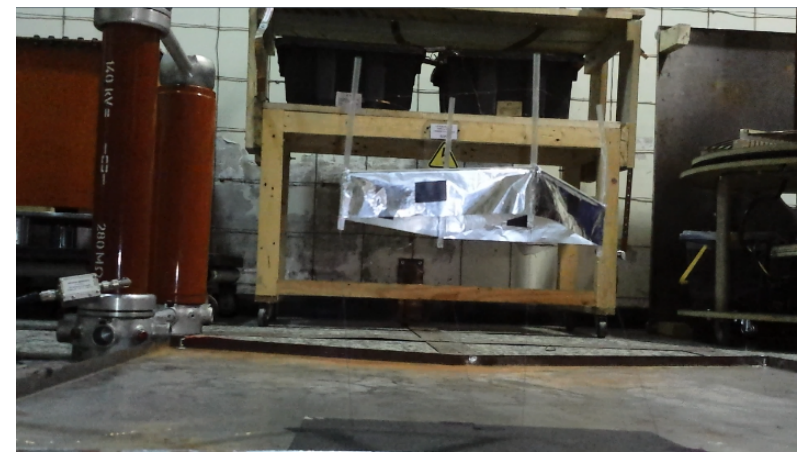

Figura 9. Sistema de puesta a tierra homogéneo.

\subsection{Levitación de los prototipos}

En la tabla 4 se presentan los resultados obtenidos al aplicar una tensión a los prototipos en corriente alterna y directa.

Tabla 4. Equipos de alta tensión.

\begin{tabular}{ccc}
\hline Condensador Asimétrico & Señal & Tensión $[\mathrm{kV}]$ \\
\hline 1 & AC & $30.9289 \pm 0.1103$ \\
1 & DC & $30.4451 \pm 0.0090$ \\
2 & AC & $29.8074 \pm 0.1069$ \\
1 & DC & $29.7046 \pm 0.0088$ \\
\hline
\end{tabular}

De acuerdo con la tabla 4, el condensador asimétrico 1 presentó una levitación estable a una tensión superior con respecto al condensador asimétrico 2, a pesar de que el condensador asimétrico 2 tiene más masa $(48.44 \%$ respecto al condensador asimétrico 1). Una vez superada la tensión registrada en la tabla 4, los condensadores asimétricos presentaron inestabilidad y arcos eléctricos, debido a que se supera la rigidez dieléctrica del aire (ver figura 10).

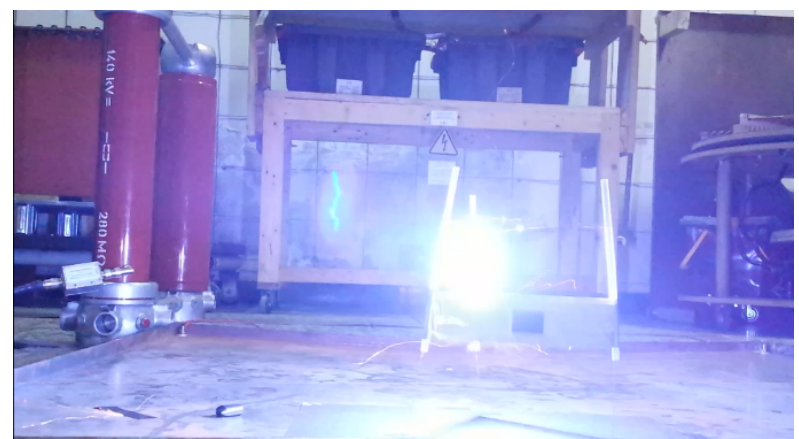

Figura 10. Presencia de arco eléctrico en el condensador asimétrico 1 en DC.

\subsection{Ensayos adicionales}

A continuación se presentan tres modelos adicionales, con el fin de observar si levitan con configuraciones diferentes a las planteadas inicialmente.
Teniendo presente la teoría de la propulsión de Thomas Townsend, de 1960, [3] se construyó un capacitor asimétrico cuadrado (ver figura 11) que contaba con las características, en principio, de un transductor electro-cinético.

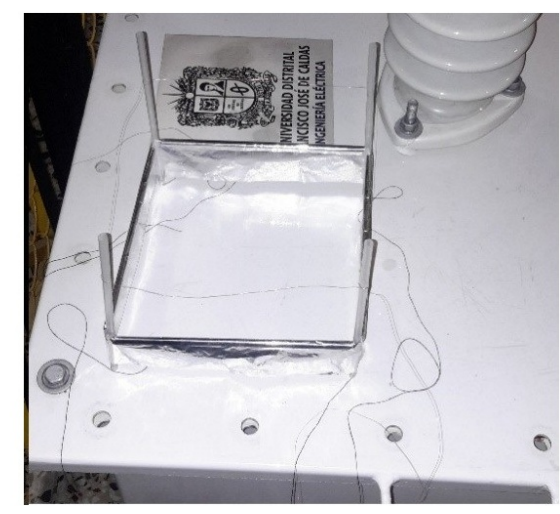

Figura 11. Capacitor asimétrico cuadrado.

Este capacitor tenía una masam $=3.95 \pm 0.01$ $\mathrm{g}$, se sometió a una tensión de $30 \mathrm{kV}$ en corriente alterna y directa, pero no presentó levitación; además, al someterlo a una tensión superior se rompió la rigidez dieléctrica del aire.

Por otra parte, se construyeron dos condensadores simétricos, con el propósito de comprobar que los electrodos de los prototipos deben ser asimétricos para levitar.

El condensador simétrico 1 tiene las mismas dimensiones que el condensador asimétrico 1 ; sin embargo, en lugar del electrodo de aluminio va uno de cobre y sus soportes son de madera de balso, puesto que con los tubos de polipropileno el prototipo se deformaba y no era estable para los ensayos.

También, se construyó un segundo condensador simétrico, con la diferencia, respecto al primero, de que los electrodos positivos y negativos eran de aluminio.

Al aplicar una tensión a los prototipos en corriente alterna y directa no se evidenció elevación; sin embargo, el condensador simétrico 1 presentó una vibración, pero al aumentar el nivel de tensión se rompe la rigidez dieléctrica del aire; ambos prototipos se sometieron a una tensión máxima de 40 $\mathrm{kV}$. 


\section{Análisis y modelado con el programa computacional Tracker}

Con el fin de observar el comportamiento de levitación, se llevó a cabo el estudio de la cinemática del sistema para ambos prototipos con el programa computacional Tracker.

\subsection{Análisis de levitación de los condensadores asimétricos}

Los experimentos realizados presentaron un movimiento predominante en el eje $\mathrm{y}, \mathrm{y}$ en el eje $\mathrm{x}$, un movimiento mínimo, razón por la cual se realizaron los análisis en el sentido vertical del movimiento.

Como se observa en la figura 12 , se crearon modelos cinemáticos y dinámicos a partir de puntos medidos en la trayectoria del condensador asimétrico.

En el programa computacional Tracker se ubicó una vara calibradora correspondiente a $\operatorname{los} 4 \mathrm{~cm}$ de la altura del electrodo negativo y se colocó un foco rastreador en el cuadro azul del electrodo negativo, como se puede apreciar en la figura 12; de esta manera, cuando el condensador asimétrico se elevó, el programa computacional registró el desplazamiento y el tiempo, los cuales permitieron el cálculo de la velocidad y la aceleración [14].

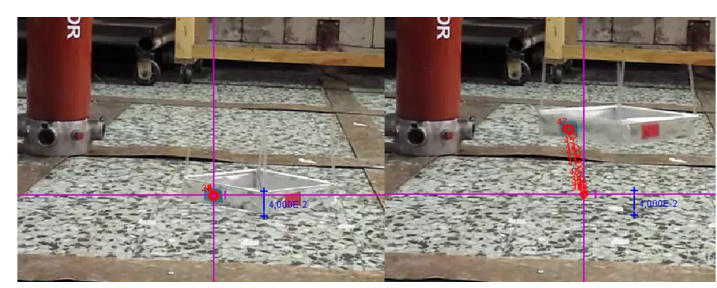

Figura 12. Simulación para obtener la aceleración y velocidad del condensador asimétrico.

Las curvas de velocidad y posición se modelaron con ecuaciones polinómicas de primer y segundo grado, respectivamente, obteniendo un coeficiente de determinación superior en los resultados finales a 0.99 en cuanto a posición y 0,88 en cuanto a velocidad, lo cual indica que la mayoría de los puntos de la señal son representados por la ecuación estimada.

A continuación se presentan los primeros resultados obtenidos a partir del análisis y modelado en el programa computacional Tracker.

En la figura 13 se observa la posición en la primera configuración, con un sistema de puesta a tierra no homogéneo.

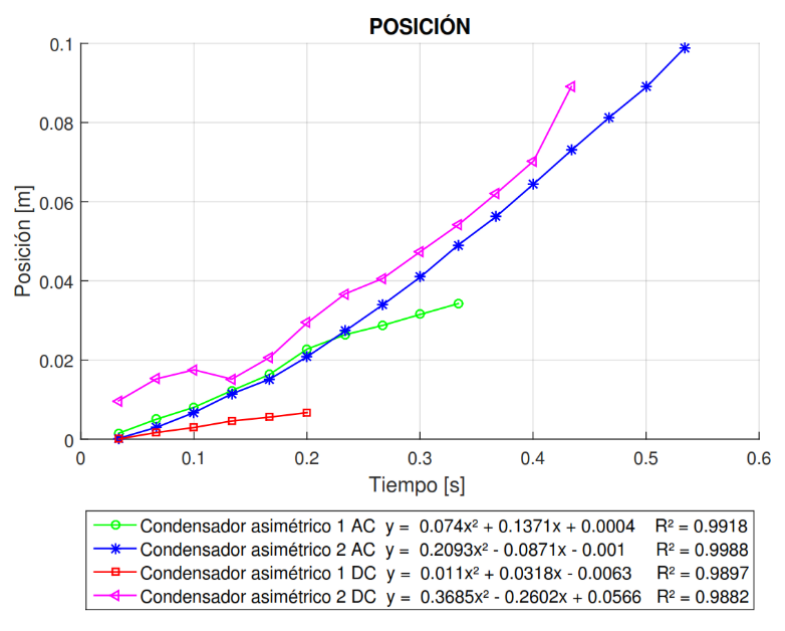

Figura 13. Curvas de posición de cada condensador asimétrico en AC y DC con el campo no homogéneo.

Para obtener el coeficiente de determinación $\mathrm{R}^{2}$ [15], en las curvas de velocidad (ver figura 14 y figura 16) se usan las ecuaciones para coeficiente de determinación $\mathrm{R}^{2}$.

Se calculó el coeficiente de determinación $\mathrm{R}^{2}$, obteniendo un coeficiente de determinación superior en los resultados finales a 0.98 y 0.89 para las curvas de posición y velocidad, respectivamente (ver figuras 14 y 16), lo cual indica que la mayoría de los puntos de la señal son representados por la ecuación estimada.

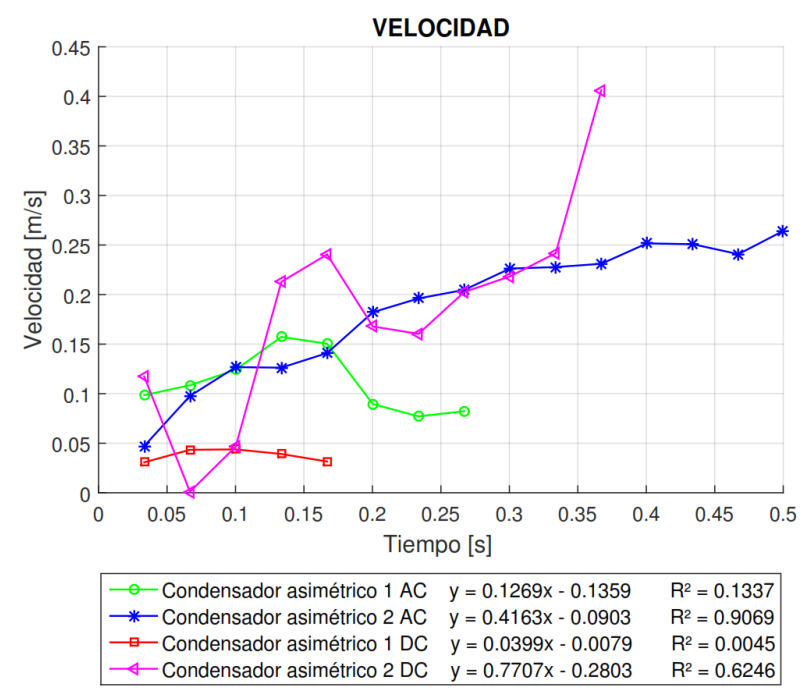

Figura 14. Curvas de velocidad de los dos condensadores asimétricos en AC y DC (sistema de puesta a tierra no homogéneo).

Para tener un sistema de puesta a tierra homogéneo y adecuado para el experimento se hizo el arreglo que se evidencia en la figura 9, el cual se utilizó para los dos modelos. 
De acuerdo con la figura directa, los dos modelos levitaron; sin embargo, la levitación no fue estable, y el modelo 2 presentó mayor aceleración, en comparación con el modelo 1.

Como se observa en la figura 15 , se mejora la estabilidad de la levitación electromagnética de los prototipos en comparación con la figura 13, con el sistema de puesta a tierra homogeneizada.
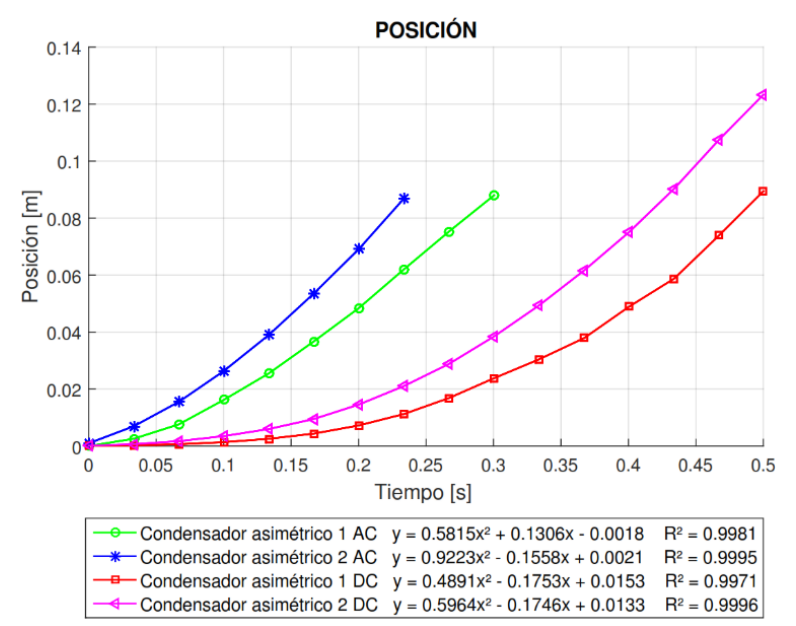

Figura 15. Curvas de posición de cada condensador asimétrico en AC y DC.
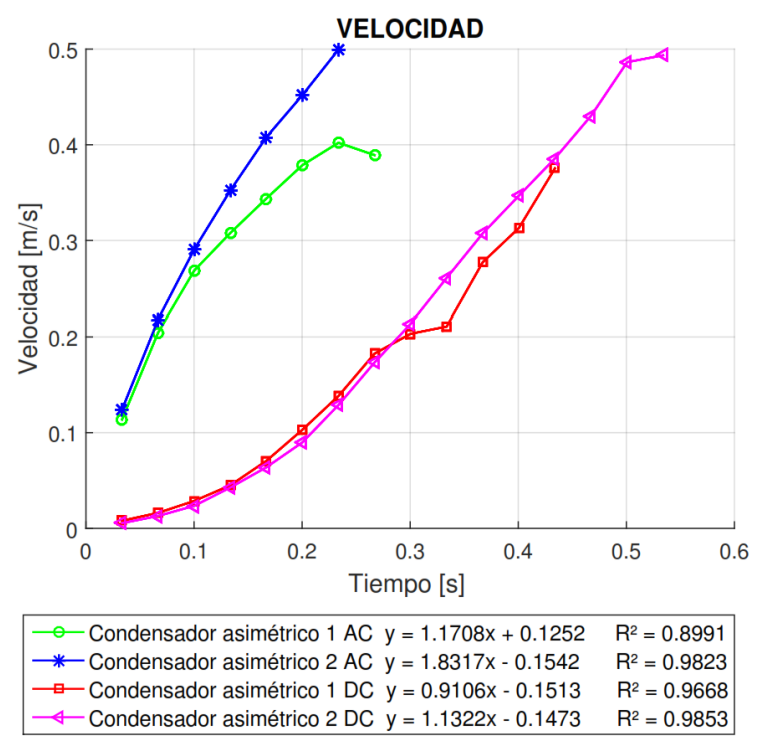

Figura 16. Curvas de velocidad de cada condensador asimétrico en AC y DC.

De acuerdo con la figura 16, la aceleración que presentaron los condensadores es mayor en un 40,6\% para el condensador asimétrico 1 en AC respecto de la señal en DC, y en un $61,7 \%$ para el condensador asimétrico 2 en AC en comparación con la señal en DC.
Un condensador en corriente continua se comporta como un circuito abierto, mientras que un condensador en corriente alterna (cuando la frecuencia tiende a infinito) tiende a comportarse como un corto circuito [16]. En otras palabras, el condensador asimétrico presenta menos impedancia en corriente alterna que en corriente directa.

Por otra parte, el condensador asimétrico 2 presentó mayor aceleración, puesto que el volumen de la geometría implica mayor captación de aire ionizado; además, requiere $3.12 \%$ menos de tensión (ver tabla 4) para obtener una levitación estable.

\subsection{Fuerza de levitación de los condensadores asimétricos}

Al obtener los valores de aceleración (el coeficiente que acompaña la variable en cada ecuación de velocidad, ver figura 16) se procedió a hallar el valor de la fuerza que presentaron los condensadores asimétricos al elevarse, como se puede apreciar en la figura 17.

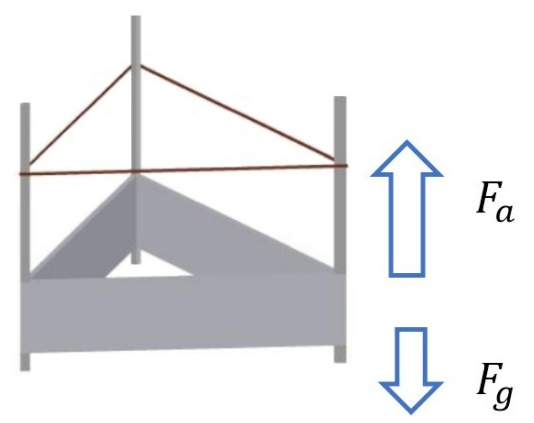

Figura 17. Diagrama de fuerzas en un capacitor asimétrico sometido a alta tensión.

$$
F_{a}=F_{o}+F_{g}
$$

Donde:

$F_{a}$ : fuerza aplicada del condensador asimétrico

$F_{o}$ : fuerza neta

$F_{g}$ : fuerza gravitacional

$$
F_{o}=m a
$$

Donde:

$a$ : aceleración obtenida del programa computacional Tracker

$m$ : masa del condensador asimétrico

$$
F_{g}=m g
$$

Donde:

$g$ : gravedad terrestre $\left(9,77 \mathrm{~m} / \mathrm{s}^{2}\right)$ [17] 
$m$ : masa del condensador asimétrico

Resolviendo la ecuaciones 6,7 y 8 se obtuvieron los resultados consignados en la tabla

Tabla 5. Fuerza de los condensadores asimétricos.

\begin{tabular}{cccc}
\hline Señal & Fuerza neta & $\begin{array}{c}\text { Fuerza } \\
\text { gravitacional }\end{array}$ & $\begin{array}{c}\text { Fuerza } \\
\text { aplicada del } \\
\text { lifter }\end{array}$ \\
\hline \multicolumn{4}{c}{ Condensador asimétrico 1 } \\
\hline \multirow{2}{*}{ AC } & $4.12 \mathrm{mN} \pm$ & $31.4 \mathrm{mN} \pm$ & $35.5 \mathrm{mN} \pm$ \\
& $5.80 \%$ & $0.310 \%$ & $0.947 \%$ \\
DC & $2.93 \mathrm{mN} \pm$ & $31.4 \mathrm{mN} \pm$ & $34.4 \mathrm{mN} \pm$ \\
& $3.26 \%$ & $0.310 \%$ & $0.593 \%$ \\
\hline \multicolumn{4}{c}{ Condensador asimétrico 2} \\
AC & $8.75 \mathrm{mN} \pm$ & $46.7 \mathrm{mN} \pm$ & $55.4 \mathrm{mN} \pm$ \\
& $1.97 \%$ & $0.209 \%$ & $0.488 \%$ \\
DC & $5.41 \mathrm{mN} \pm$ & $46.7 \mathrm{mN} \pm$ & $52.1 \mathrm{mN} \pm$ \\
& $1.67 \%$ & $0.209 \%$ & $0.361 \%$ \\
\hline \multicolumn{4}{c}{}
\end{tabular}

Como se observa en la tabla 5 , los condensadores en corriente alterna indujeron mayor fuerza para su elevación, en comparación con la señal en corriente directa, siendo un $3.34 \%$ y $6.01 \%$ mayor en corriente alterna para el condensador asimétrico 1 y 2 , respectivamente, que en corriente directa.

Los ensayos en corriente alterna presentaron mayor aceleración y fuerza, además de menor tensión aplicada para llegar a una levitación parcialmente estable.

Durante los ensayos experimentales se observó mayor estabilidad en corriente directa que en corriente alterna, e incluso se alcanzó mayor altura, como se aprecia en la tabla 6.

Tabla 6. Altura de levitación alcanzada por los condensadores asimétricos 1 y 2 .

\begin{tabular}{ccc}
\hline Señal & $\begin{array}{c}\text { Análisis de las curvas } \\
\text { cinemáticas }\end{array}$ & $\begin{array}{c}\text { Altura máxima } \\
\text { alcanzada }\end{array}$ \\
\hline \multicolumn{3}{c}{ Condensador asimétrico 1} \\
\hline AC & $8.7 \pm 0.1 \mathrm{~cm}$ & $9.1 \pm 0.1 \mathrm{~cm}$ \\
DC & $8.9 \pm 0.1 \mathrm{~cm}$ & $9.3 \pm 0.1 \mathrm{~cm}$ \\
\hline \multicolumn{3}{c}{ Condensador asimétrico 2} \\
\hline AC & $8.6 \pm 0.1 \mathrm{~cm}$ & $11.7 \pm 0.1 \mathrm{~cm}$ \\
DC & $12.3 \pm 0.1 \mathrm{~cm}$ & $12.3 \pm 0.1 \mathrm{~cm}$ \\
\hline
\end{tabular}

Por otra parte, el condensador asimétrico vibró y emitió un sonido similar a un torrente de aire soplando al capacitor asimétrico, al estar sometido a la señal de corriente alterna. En cuanto a las pruebas en corriente continua, el capacitor asimétrico no emitió ningún sonido.

\section{Análisis y modelación con el programa compu- tacional Comsol Multiphisycs}

En el programa computacional Comsol Multiphisycs se desarrolló la modelación y simulación de los prototipos, con las físicas de campo eléctrico y magnético, con el objetivo de observar el comportamiento del condensador asimétrico en presencia de estos campos.

Suministrando energía a los prototipos a una tensión aproximada de $30 \mathrm{kV}$ en AC y DC, se presenta un campo eléctrico intenso en las proximidades del cable, ionizando el aire y, de esta manera, permitiendo que los iones se desplacen hacia el electrodo de tierra colisionando con las moléculas de aire neutras en su camino, y como resultado de la interacción del campo eléctrico con las moléculas o partículas ionizadas se genera un flujo electrohidrodinámico, permitiendo la elevación del condensador asimétrico [18].

\subsection{Modelación preliminar}

Para observar más detalladamente el comportamiento de las líneas de campo eléctrico, se hizo una modelación del condensador asimétrico con un corte trasversal, teniendo presente la placa que se encuentra sobre el sistema de puesta tierra.

Como se observa en la figura 18 , el electrodo positivo se encuentra a un potencial de $30 \mathrm{kV}$; el terminal negativo y la placa equipotencial se encuentran referidos a tierra.

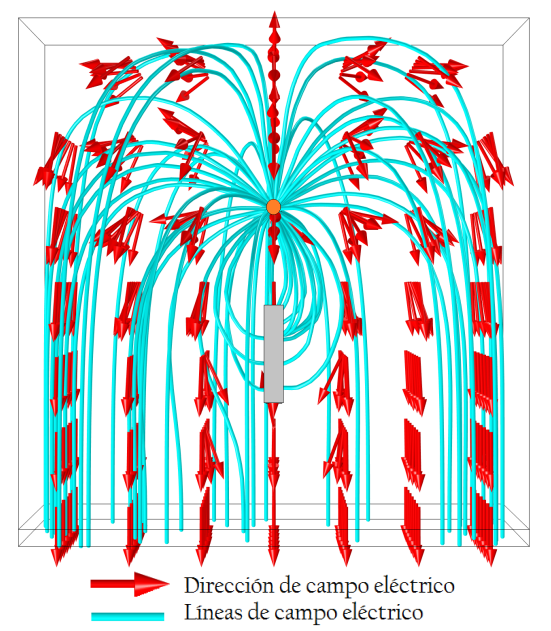

Figura 18. Simulación de campo eléctrico desde una vista transversal del condensador asimétrico 1 .

De acuerdo con la Figura 18, las líneas de campo eléctrico del electrodo positivo son dirigidas radial- 
mente hacia afuera, y finalmente llegan al electrodo negativo, y las que se encuentran más alejadas llegan a la placa equipotencial, por lo cual, las líneas que están bien dirigidas hacia el electrodo negativo facilitan la migración de los electrones hacia el electrodo positivo, formando moléculas cargadas.

Así mismo, el medio dieléctrico (aire) tiende a ionizarse; estas moléculas son repelidas fuertemente por el electrodo positivo, sin embargo, son atraídas hacia el electrodo opuesto, generando de esta manera una nube de iones que se mueven hacia el electrodo negativo, colisionando con moléculas neutras circundantes del aire, presentando así un empuje o propulsión electrodinámica [19].

\subsection{Análisis y modelado en corriente alterna}

A continuación se presenta la distribución del campo eléctrico y magnético en los prototipos modelados.

Como se observa en la figura 19, las líneas de campo eléctrico salen del electrodo positivo hacia el electrodo negativo, y se presencia un efecto de borde, por lo cual las demás líneas se van hacia la lámina, debido a que se encuentra referida a tierra.

Por otra parte, como el campo eléctrico es variante en el tiempo, también se presenta un campo magnético que es perpendicular a las líneas de campo eléctrico que se encuentran dirigidas hacia el electrodo opuesto.

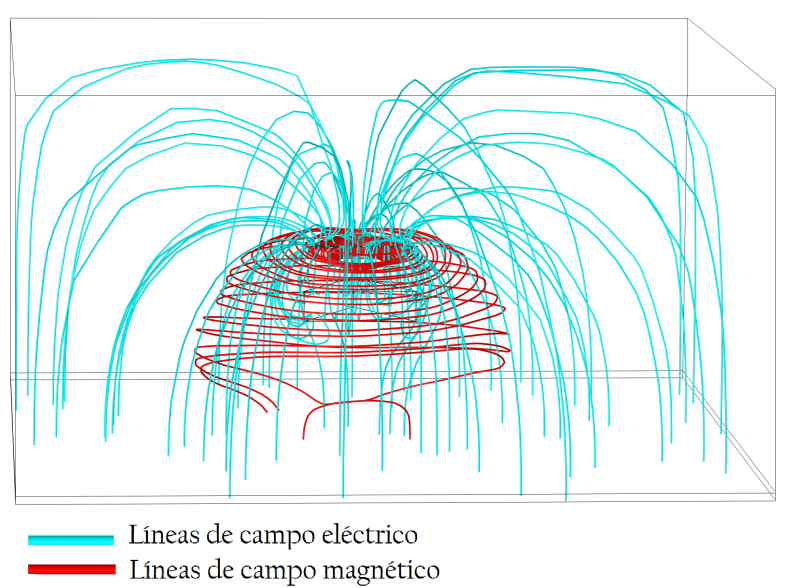

Figura 19. Simulación de campo eléctrico y magnético de condensador asimétrico 1.

El comportamiento que presenta el condensador asimétrico 2 (ver figura 20) ante un campo variante en el tiempo es similar al del condensador asimétrico 1 , con la diferencia de que los campos son más intensos; además, la concentración de campo magnético tiende a ser más uniforme, debido a una mayor captación de campo por el tipo de geometría.

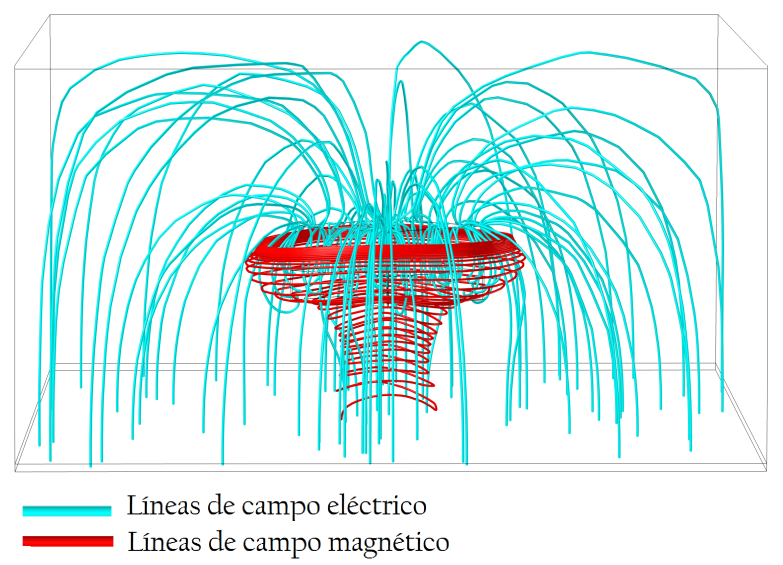

Figura 20. Simulación de campo eléctrico y magnético de condensador asimétrico 2 .

\subsection{Análisis de campo eléctrico}

Para observar la magnitud de campo eléctrico y magnético, se obtiene una gráfica del programa computacional Comsol Multiphysics a partir de los modelos simulados.

El potencial eléctrico se encuentra en el electrodo positivo, presentando mayor intensidad de campo eléctrico, el cual disminuye hasta llegar al electrodo negativo, permitiendo observar que en el medio dieléctrico está presente la acción de un campo eléctrico (ver figura 21 y 22).

La diferencia de intensidad de campo eléctrico entre los modelos es de un $70.37 \%$ presentando mayor intensidad de campo en el condensador asimétrico 2.

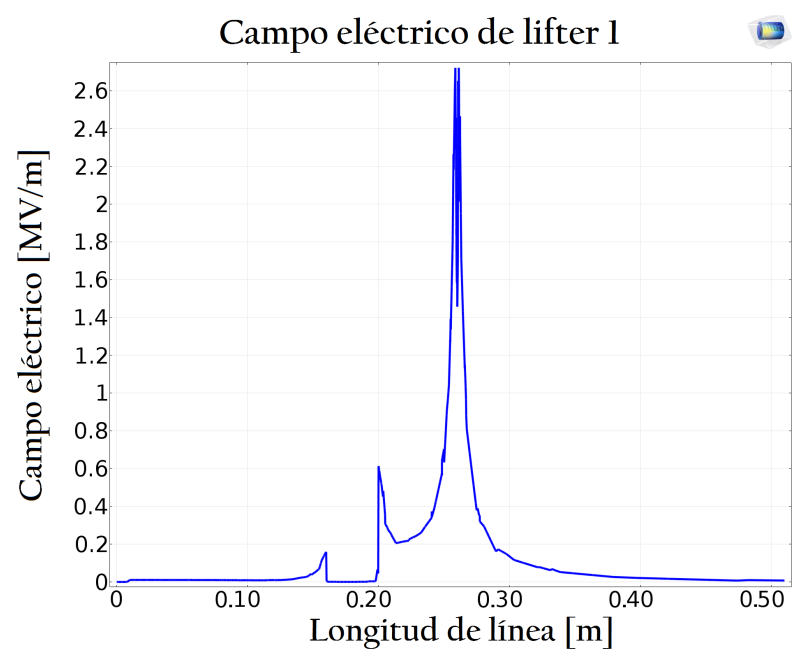

Figura 21. Curva de campo eléctrico de condensador asimétrico 1. 


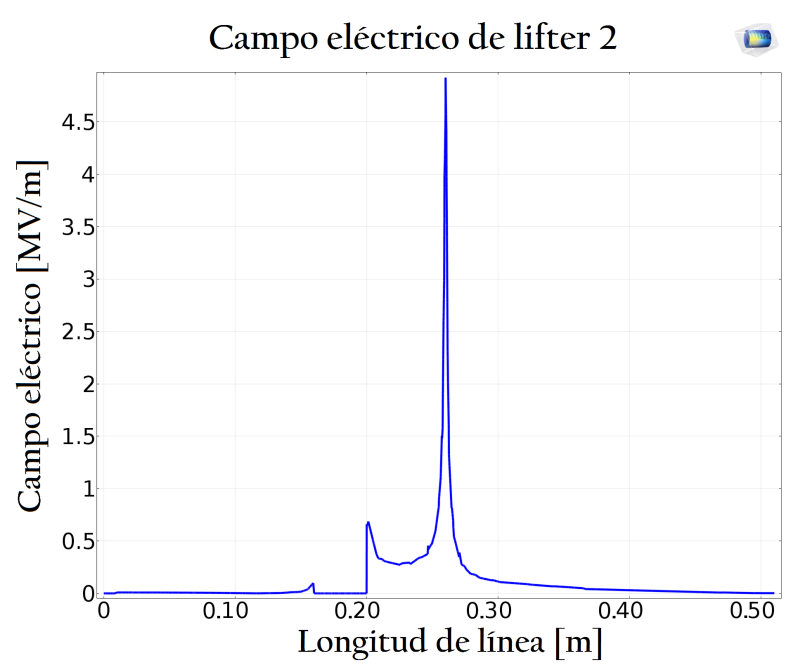

Figura 22. Curva de campo eléctrico de condensador asimétrico 2 .

\subsection{Análisis de campo magnético}

Debido a la variación en el tiempo de campo eléctrico, se presenta un campo magnético que es de menor intensidad, verificando de esta manera que, en ausencia del campo magnético, el condensador asimétrico actúa por la interacción de fuerzas eléctricas, y en presencia de una variación de campo magnético, el aporte tiende a ser mínimo.

Como se observa en las figuras 23 y 24 , se presenta una disminución de campo magnético desde el electrodo positivo hacia el electrodo negativo, presentando mayor intensidad el modelo del condensador asimétrico 2, siendo el $47.36 \%$ mayor al condensador asimétrico 1 .

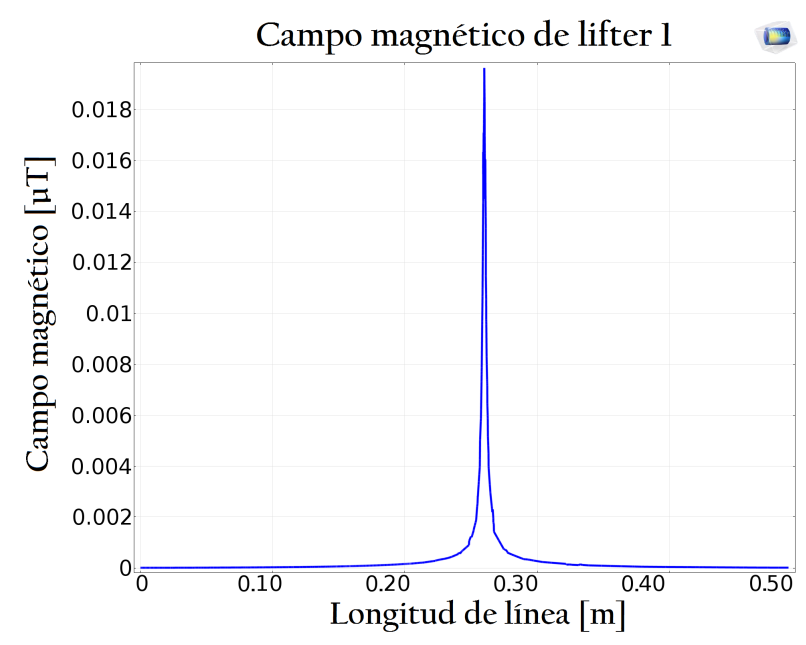

Figura 23. Curva de campo magnético de condensador asimétrico 1.

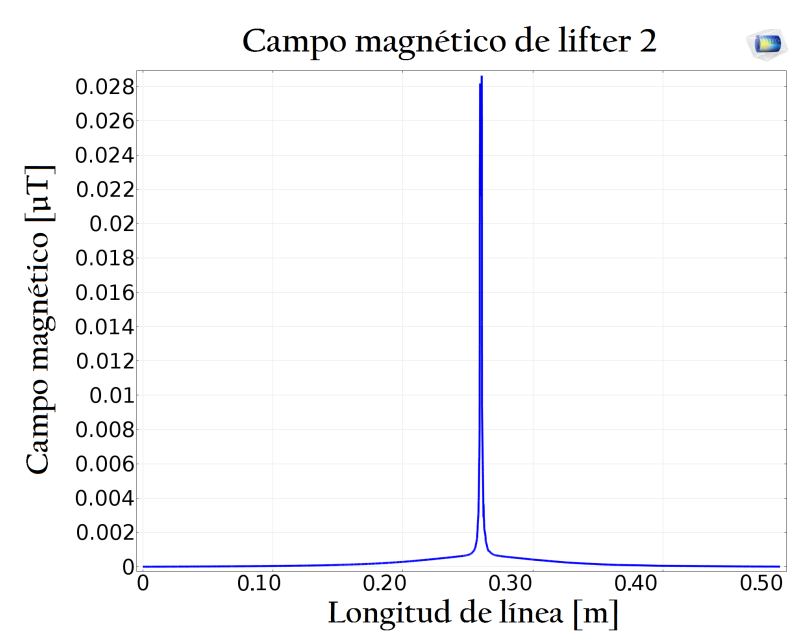

Figura 24. Curva de campo magnético de condensador asimétrico 2.

\subsection{Análisis y modelado en corriente directa}

El campo eléctrico que se presenta en los condensadores asimétricos 1 y 2 (ver figura 25 y 26) no es uniforme, puesto que la mayoría de las líneas de campo tienden a presentar el efecto de borde; sin embargo, se puede observar cierta cantidad de líneas que van desde el electrodo positivo hacia el electrodo opuesto; el desbordamiento de las demás líneas permite que los iones no se desplacen hacia el electrodo opuesto, lo cual facilita una ionización del aire alrededor del prototipo.

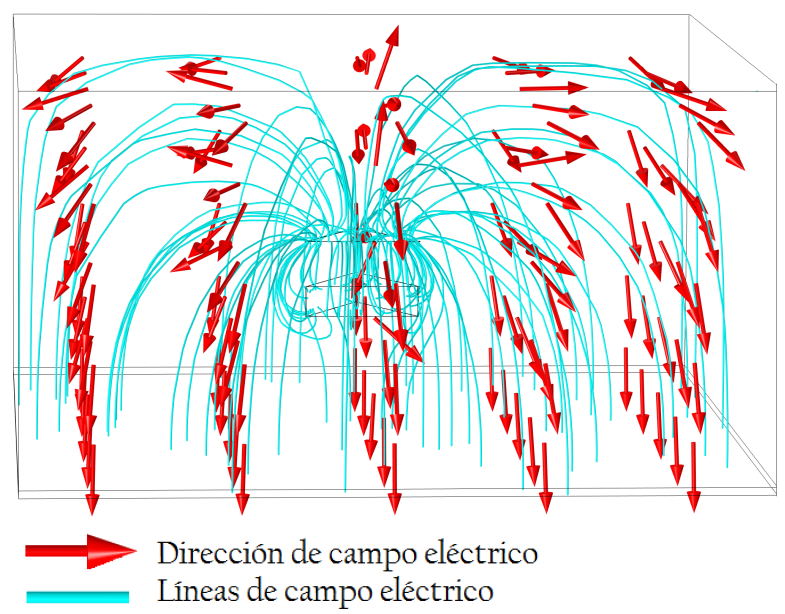

Figura 25. Simulación de campo eléctrico de condensador simétrico 1.

Como se observa en la figura 26, debido a la geometría de los dos triángulos equiláteros que conforman el condensador asimétrico 2, este presenta mayor intensidad de campo eléctrico, lo cual presume mayor propulsión electrodinámica. 


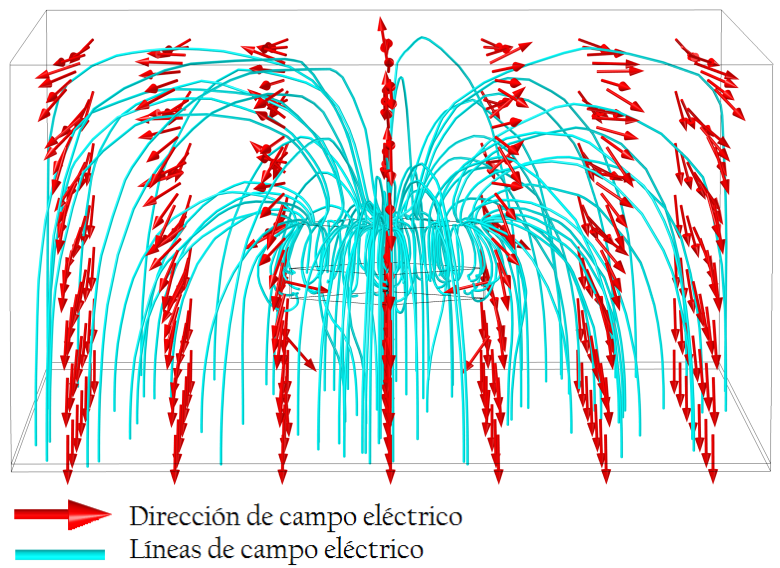

Figura 26. Simulación de campo eléctrico de condensador asimétrico 2 .

\section{Imágenes termográficas}

A continuación se presentan unas imágenes, tomadas con la cámara de termografía FLIR, con el objetivo de observar la distribución y variación térmica en los prototipos de condensador asimétrico.

Para realizar una correcta medición de termografía fue necesario tomar como referencia la temperatura de un fragmento de cinta adhesiva con una emisividad de 0.95 , debido a la baja emisividad de la superficie reflectiva del aluminio.

\subsection{Condiciones iniciales}

Se obtuvieron imágenes con la cámara de termografía antes de aplicar tensión a los condensadores asimétricos (ver figuras 27 y 28).

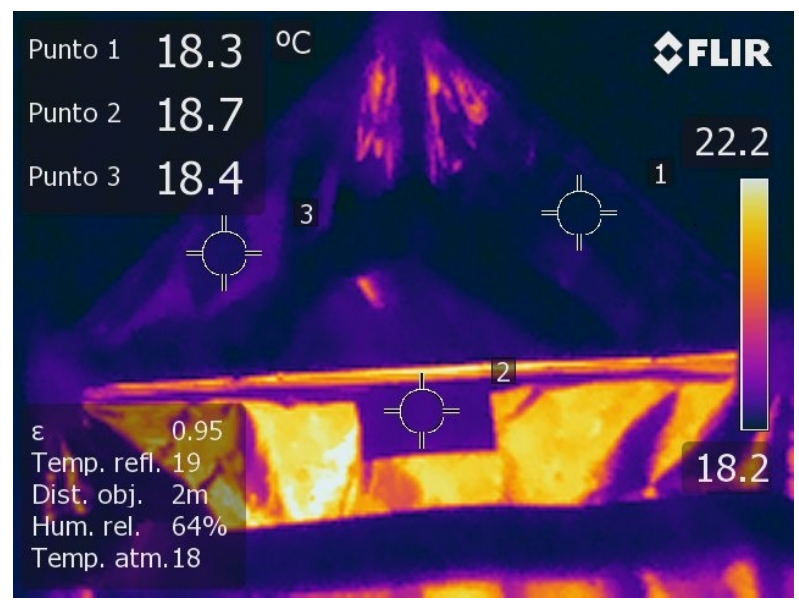

Figura 27. Distribución térmica del condensador asimétrico 1.

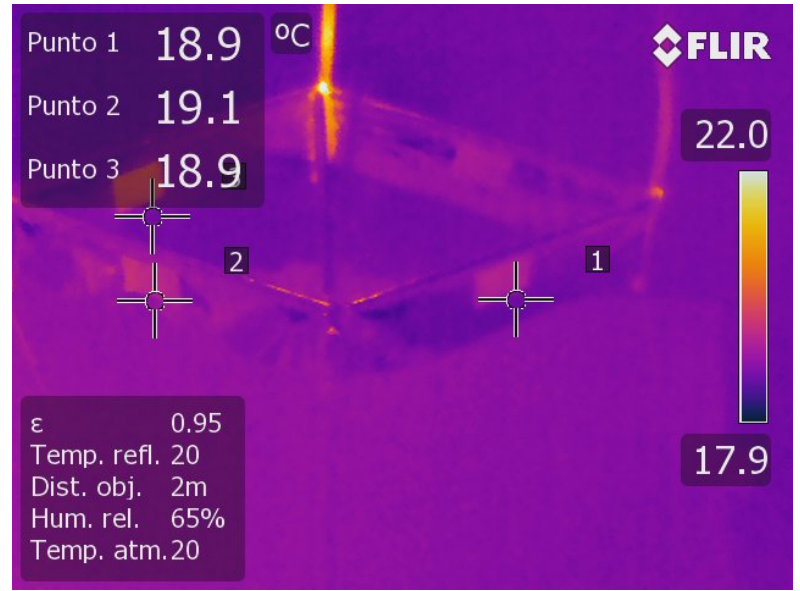

Figura 28. Distribución térmica del condensador asimétrico 2.

\subsection{Tensión aplicada}

Durante la aplicación de una tensión eléctrica se obtuvieron muestras termográficas en corriente alterna y directa de los condensadores asimétricos.

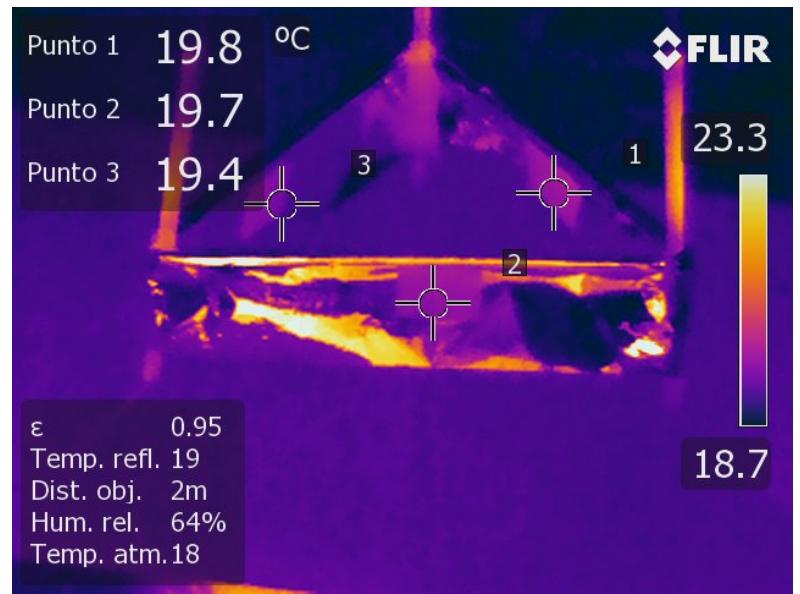

Figura 29. Distribución térmica de condensador asimétrico 1 (tensión aplicada en AC).

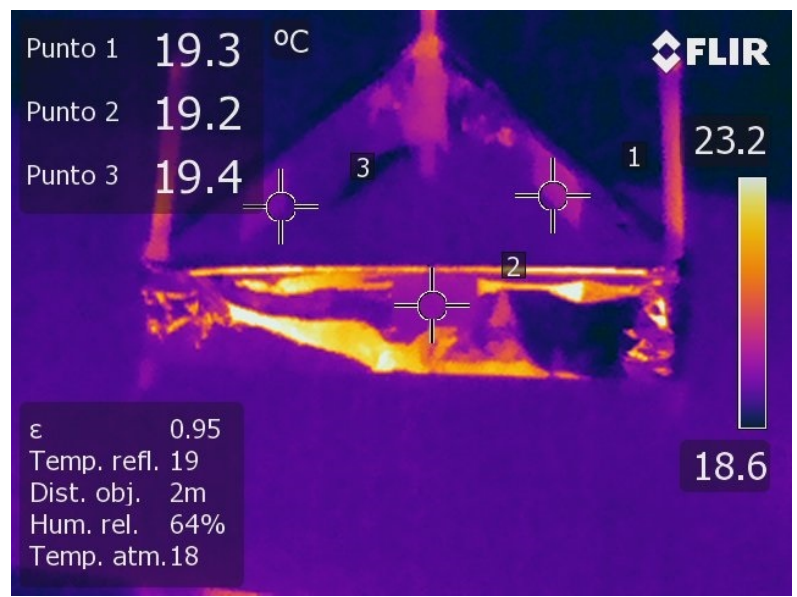

Figura 30. Distribución térmica del condensador asimétrico 1 (tensión aplicada en DC). 


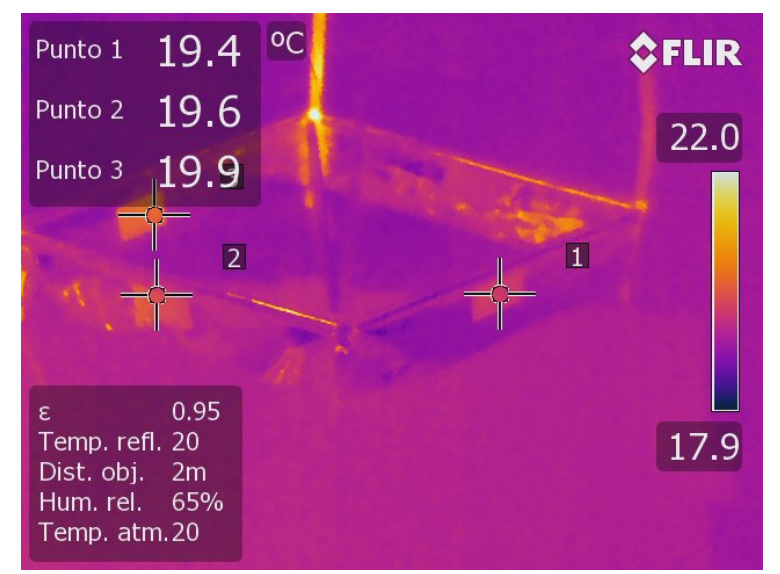

Figura 31. Distribución térmica del condensador asimétrico 2 (tensión aplicada en AC).

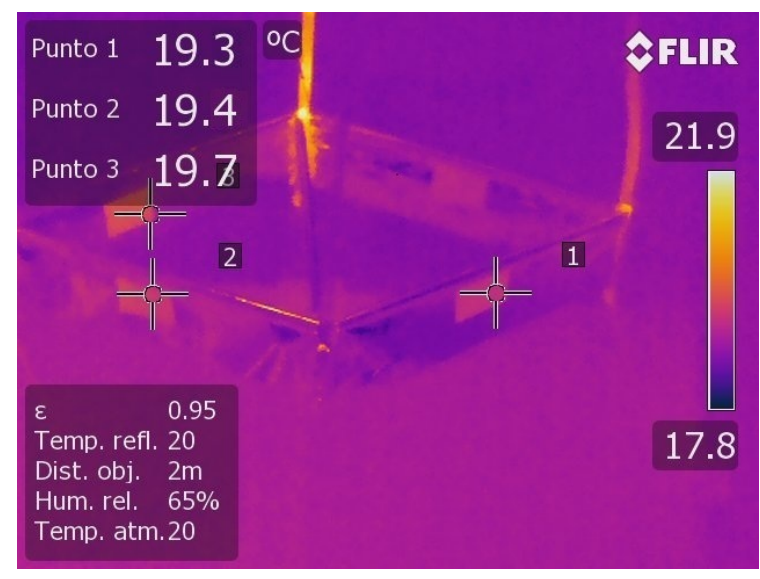

Figura 32. Distribución térmica de condensador asimétrico 2 (tensión aplicada en DC).

En la tabla 7 se presenta un resumen de los datos obtenidos en el estudio de termografía; en él se observan los tres puntos de medición para cada prototipo.

La variación de temperatura que presentaron los condensadores asimétricos con cada tipo de señal se debe al movimiento de moléculas ionizadas y electrones libres, el cual causa colisiones entre ellas y disipa una cantidad de energía [20] que se puede apreciar en un cambio de temperatura al aplicar tensión a los condensadores asimétricos a un alto potencial (30 $\mathrm{kV})$.

Por otra parte, en corriente alterna se presentó una variación de temperatura del $2 \%$ más que en corriente directa, debido a que las moléculas ionizadas y los electrones libres se acercan y se alejan de la fuente del campo eléctrico continuamente [20], causando de esta manera mayor cantidad de colisiones y, por lo tanto, mayor liberación de energía, representada en una variación de temperatura.
Tabla 7. Altura de levitación alcanzada por los condensadores asimétricos 1 y 2 .

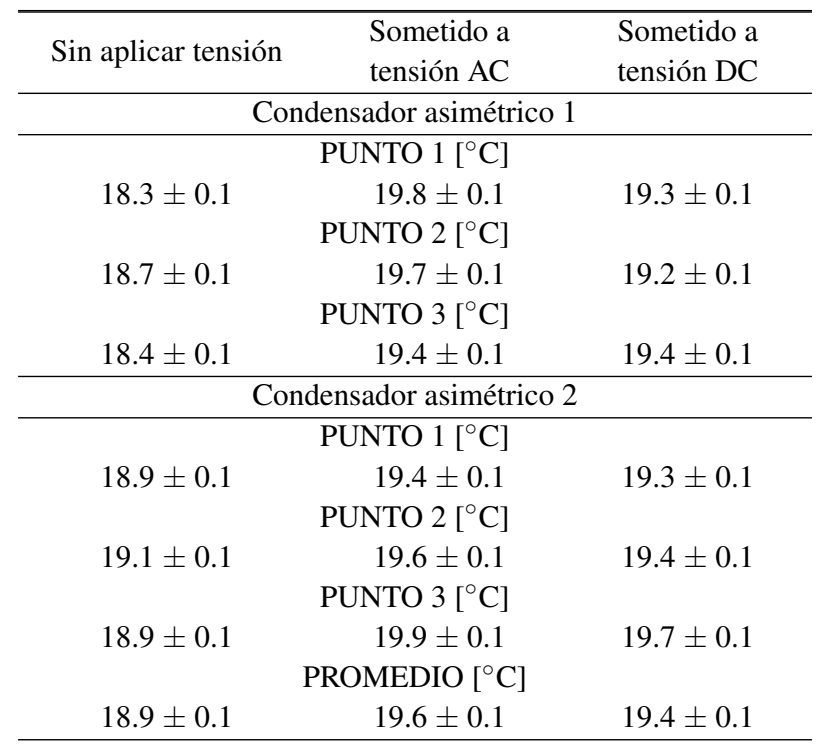

\section{Conclusiones}

El condensador asimétrico con geometrías triangulares facilita una propulsión, permitiendo que los prototipos leviten, a diferencia de una geometría cuadrada, que no levitó.

La lámina equipotencializadora utilizada para mejorar el sistema de puesta a tierra garantizó un comportamiento suave de las líneas de campo eléctrico, debido a que se homogeneiza el electrodo negativo del laboratorio de alta tensión, el cual es una cuadricula de láminas de cobre que son comparables con las dimensiones del condensador asimétrico.

El estudio en corriente alterna da como resultado que los condensadores asimétricos presentan mayor fuerza sustentadora al momento de levitar; además, el condensador asimétrico 2, en corriente alterna y directa, presentó una levitación con mayor fuerza y mejor estabilidad, a diferencia del condensador asimétrico 1 .

Los ensayos experimentales evidenciaron que los condensadores asimétricos presentan una levitación más estable en corriente directa que en corriente alterna, debido a que el campo eléctrico no varía en el tiempo, a pesar de que los prototipos también levitaron en corriente alterna. 
Comparando el condensador asimétrico 1 y 2 , es visible un incremento de fuerza al cambiar de diseño, presentando el modelo 2 mayor masa y mayor aceleración; sin embargo, no se presentó un incremento lineal. Se considera que este incremento en la fuerza es debido a la geometría del condensador asimétrico 2; un triángulo equilátero más en la geometría aumenta la fuerza, permitiendo levantar más masa que la correspondiente al condensador asimétrico de un solo triángulo equilátero.

Las simulaciones realizadas en el programa computacional Comsol Multiphisycs permitieron observar la intensidad del campo eléctrico y magnético que presentan los prototipos, siendo el campo eléctrico el más relevante en el fenómeno de la levitación.

Las imágenes de termografía evidenciaron un cambio mínimo de temperatura al aplicar tensión a los prototipos, por lo tanto, la teoría de los materiales ablativos, la cual enuncia que los electrodos presentan altas temperaturas hasta llegar a su deformidad en el momento de la levitación, queda descartada.

De las teorías planteadas anteriormente, que explican el efecto Biefield-Brown, la más viable, de acuerdo con los resultados obtenidos, es la presencia de ionización del medio dieléctrico (aire), provocando la transferencia del momentum de los electrones en las líneas de campo al aire, lo que lleva al empuje que permite la levitación.

\section{Trabajo a futuro}

En el Laboratorio de Alta Tensión solo se desarrollaron ensayos experimentales en dos frecuencias: 0 y $60 \mathrm{~Hz}$; es factible hacer ensayos en altas frecuencias, con el fin de analizar el comportamiento que presentarían los condensadores asimétricos.

Debido a los inconvenientes presentados por el sistema de puesta a tierra del Laboratorio de Alta Tensión, el cual no era homogéneo, se podría realizar un dispositivo con una fuente aparte (una tierra flotante) y realizar ensayos experimentales en el laboratorio para verificar posibles interferencias de un circuito ajeno al sistema de propulsión iónica.

Al ubicar un triángulo equilátero más en la geometría del condensador asimétrico se presenta un aumento de fuerza, que no es lineal. Es viable hacer ensayos con geometrías que impliquen más triángulos equiláteros, para hallar un modelado estadístico que estime en qué proporción aumenta la fuerza al colocar más triángulos, con la finalidad de comprobar si es posible cambiar los materiales por unos más fuertes y resistentes para un estudio a futuro de transporte con condensadores asimétricos.

\section{Agradecimientos}

A Dios y nuestros seres queridos. Al profesor Omar Bohórquez que nos brindo su asesoría durante la investigación, a Alexandra Martínez, Alexis Ladino y a los docentes que hicieron parte de nuestra formación académica en la Universidad Distrital Francisco José de Caldas.

\section{Referencias}

[1] J. W. Cambell, Apparatus for generating thrust using a two dimensional asymmetrical capacitor module, U.S Patent 6414 493, June 25, 2002.

[2] F. X. Canning, C Melcher, E Winet, Asymmetrical Capacitors for Propulsion, Nasa, Institute for Scientific Research, Inc., Fairmont, West Virginia, CR - 2004 - 213312, Oct 2004.

[3] T. T. Brown, Electrokinetic transducer.U.S Patent 3018 394, Jan 23, 1962.

[4] S. Fech, 8 ways magnetic levitation can shape the future [online]. Estados Unidos, 2012. Disponible en: http://www.popularme chanics.com/technology /infrastructure/g89/8ways-magnetic-levitation-could-shape-thefuture/?slide $=1$.

[5] L. Zhao, K. Adamiak, "Effects of EHD and External Airflows on Electric Corona Discharge in Point-Plane/Mesh", Proc IEEE Transactions on industry, vol. 45, no. 1, pp. 1 y 6, Feb 2009.

[6] A. A. Martins, M. J. Pinheiro, "Modeling of an EHD corona flow in nitrogen gas using an asymmetric capacitor for propulsion," Proc ScienceDirect, pp. 1, 2011.

[7] J. Rincón, I. Amaya, "Assymetrical capacitor: experiments and modeling" Proc Ingenierias Universidad de Medellín vol. 11, n.ž 20, pp. 5-10, 2012.

[8] J. Primas, M. Malík, D. Jasíková, y V. Kopecký, "The Influence of Electrode Heating On the Force Generated On a High Voltage Capacitor with Asymmetrical Electrodes", Proc Interna- 
tional Science Index, vol. 6, no. 7, pp. 1 y 3, 2012.

[9] V. G Souza, M. Cattani and A. Vannucci, "Understanding the Physics of the Asymmetric Flying Capacitor", Proc. IEEE, pp. 1, 2014.

[10] C. Howell, Electrohydrodynamic Thrusters: An Examination of the Biefeld-Brown Effect and its Influence on its Surroundings, Thesis Bachelor of Science, University Honors College, Portland State University PDXScholar, Portland State University, 2014.

[11] Nasa, Summer of innovation [online]. NASA: Summer of innovation: Thermal Protection Systems Challenge, 2012 Disponible en: http://www.nasa.gov/sites/default/files/544864 main_E2_Thermal_Protection_Systems_C4.pdf.

[12] R. A. Serway,Cantidad de movimiento lineal y colisiones, En Física para ciencias e ingeniería, S. R. Cervantes, CENGAGE Learning, Mexico D.F., 2008, pp. 228-238.

[13] T. T. Brown, Electrokinetic apparatus. U.S Patent 2949 550, Aug 16, 1960.

[14] O. S. Physics, opensourcephysics.org [online]. 9 enero 2012. Disponible en http://www.compadre.org/OSP/Features/ FeatureSummary.cfm?FID=1567.
[15] S. C. Chapra, "Regresión por mínimos cuadrados", En Métodos numéricos para ingenieros, L. Campa, Mexico D.F, Mc Graw Hill, 2006, pp. 466-474.

[16] J. E. López, J. Muñoz, G. Camps, "Capacitores e Inductores", En Análisis de circuitos, Valencia: M. Simón, Universidad de Valéncia, 2006, pp. 74.

[17] A. Cárdenas, L.A. Castillo, "Interpretación de datos gravimétricos con la señal analítica 3D. Estudio de caso: Los Naranjos - Facatativá (Colombia)", Proc Boletín de Geología, vol. 35, pp. 99. 2013.

[18] L. Zhao, T. M. J. Liaw, "Thrust Origin in EHD Lifters", Proc IEEE, pp. 1, Oct 2011.

[19] J. Chu, MIT Noticias Office, "Electrohydrodynamic effect offers promise for efficient propulsion in air", Laboratory for Aviation and the Environment Massachusetts Institute of Technology, 2013. [online]. Disponible en: http://lae.mit.edu/ehd/.

[20] D. Martínez, C. Tejada, J. Hernández, Determinación del efecto corona en el ramal de una red de distribución, Ingeniería Eléctrica, Instituto Politécnico Nacional Escuela Superior de Ingeniería Mecánica y Eléctrica. Tesis de pregrado, México D.F, 2010. 